\title{
Orientation contrast sensitive cells in primate V1 a computational model
}

\author{
Tino Lourens $\cdot$ Emilia Barakova
}

Received: 6 February 2006/Accepted: 17 August 2006/Published online: 12 April 2007

(C) Springer Science+Business Media B.V. 2007

\begin{abstract}
Many cells in the primary visual cortex respond differently when a stimulus is placed outside their classical receptive field (CRF) compared to the stimulus within the $\mathrm{CRF}$ alone, permitting integration of information at early levels in the visual processing stream that may play a key role in intermediate-level visual tasks, such a perceptual popout [Knierim JJ, van Essen DC (1992) J Neurophysiol 67(5):961-980; Nothdurft HC, Gallant JL, Essen DCV (1999) Visual Neurosci 16:15-34], contextual modulation [Levitt JB, Lund JS (1997) Nature 387:73-76; Das A, Gilbert CD (1999) Nature 399:655-661; Dragoi V, Sur M (2000) J Neurophysiol 83:1019-1030], and junction detection [Sillito AM, Grieve KL, Jones HE, Cudiero J, Davis J (1995) Nature 378:492-496; Das A, Gilbert CD (1999) Nature 399:655-661; Jones HE, Wang W, Sillito AM (2002) J Neurophysiol 88:2797-2808]. In this article, we construct a computational model in programming environment TiViPE [Lourens T (2004) TiViPE-Tino's visual programming environment. In: The 28th Annual International Computer Software \& Applications Conference, IEEE COMPSAC 2004, pp 10-15] of orientation contrast type of cells and demonstrate that the model closely resembles the functional behavior of the neuronal responses of nonorientation (within the CRF) sensitive 4C $\beta$ cells [Jones HE, Wang W, Sillito AM (2002) J Neurophysiol 88:2797-2808], and give an explanation of the indirect information flow in V1 that explains the behavior of orientation contrast sensitivity. The computational model of orientation contrast cells demonstrates excitatory responses at edges near junctions that might facilitate junction detection, but the model does not reveal perceptual pop-out.
\end{abstract}

Keywords Early vision $\cdot$ V1 Orientation contrast cells $\cdot$ TiViPE simulation

T. Lourens (ه)

Philips Medical Systems Nederland B.V, Veenpluis 4-6, 5680 DA Best, The Netherlands e-mail: tino.lourens@philips.com

E. Barakova

Technical University of Eindhoven, Den Dolech 2, 5612 AZ Eindhoven, The Netherlands e-mail: e.i.barakova@tue.nl 


\section{Introduction}

Neurons in the primary visual cortex (V1) respond in well-defined ways to stimuli within their classical receptive field (CRF), but these responses can be modified by additional peripheral stimuli. The size of the periphery (non-classical surround) provides input from a larger portion of the visual scene than originally thought, permitting integration of information at early levels in the visual processing stream. Neuronal responses in V1 to static texture patterns are suppressed by textured periphery, while these cells tended to respond more strongly to a stimulus in which there was a contrast in orientation between center and surround compared to a stimulus lacking such contrast (Knierim and van Essen 1992).

Recent works indicate that neuronal surround modulation at cross-orientation, an orientation orthogonal to the preferred orientation of the CRF, might play a key role in intermediate level visual tasks, such as perceptual pop-out (Knierim and van Essen 1992; Nothdurft et al. 1999), contrast facilitation (Cavanaugh et al. 2002; Yu et al. 2002), and contextual modulation (Levitt and Lund 1997; Das and Gilbert 1999; Dragoi and Sur 2000). The strength of this contextual influence on a neuron can be predicted from a model of local connection based on simple overlap with particular features, which indicates that local intra cortical circuitry could endow neurons with a graded specialization for processing angular visual features such as corners and junctions (Sillito et al. 1995; Das and Gilbert 1999; Jones et al. 2002).

Depending on the orientation of an inner and outer grating pattern, these neuronal cells have the tendency to respond strongly to a center orientation preference or orientation contrast ${ }^{1}$ between inner and outer pattern. Neuronal output activity was enhanced in both cat and macaque primary visual cortex (V1) when, a surrounding field at a significantly different orientation $\left(30^{\circ}\right.$ or more) was added to the preferred orientation of the CRF (Sillito et al. 1995). Cells in layer 4C $\beta$, which are non-orientation sensitive within their $\mathrm{CRF}$, also show these response profiles indicating that there must be a strong feedback from other areas (within V1) that create these more complex profiles. We assume that these cells obtain feedback from complex cells in layers 2, 3, 5, and 6 of V1. The aim of this article is to setup a computational model of this type of cells which we will term orientation contrast cells, and to simulate these cells in visual programming environment TiViPE (Lourens 2004).

The article is organized as follows: Sect. 2 elaborates on the properties of non-orientation tuned cells with respect to orientation contrast, their pathway in early vision, and provides a computational model. Section 3 gives a TiViPE simulation that provides the results of this model when applied to the stimuli given by Jones et al. (2002). The article finishes with a discussion.

\section{Non-orientation tuned cells}

In primate V1 cells $94 \%$ had a response to orientation contrast stimuli that exceeded the response to the inner stimulus alone, independent from the diameter of the surround patch, while the responses were somewhat inhibitory when the orientation of the inner and outer stimuli were the same, compared to the response to the inner stimulus alone (Jones et al.

\footnotetext{
${ }^{1}$ Orientation contrast is the difference between preferred orientation of a center patch (which roughly covers the CRF) and preferred orientation of a surround patch (outside the CRF). This contrast is strongest when the center and surround orientations are orthogonal and weakest when both are the same.
} 
2002). They found that the responses of $4 C \beta$ cells could be modulated by varying both orientation of a center grating patch (inside the CRF) and a surround grating patch (outside the CRF), despite the cell's lack of orientation tuning within the CRF. Its response output was extremely sensitive to orientation differences between center and surround patches.

The LGN parvo cellular cells $(\mathrm{P})$ have center-surround shaped receptive field profiles which optimally respond to a spot of light. In a feed-forward processing stream one could expect a similar receptive field type in layer $4 \mathrm{C} \beta$. For instance, a set of center-surround profiles that are aligned in a certain way, may respond strongly to a line or bar of a specific orientation. However, such profile does not provide center orientation preference nor is it able to provide a measure for center-surround orientation contrast. The modulation of its response behavior must be caused by an indirect (feedback loop) information stream, as illustrated in Fig. 1. The local feedback connections from deep layers ( 5 and 6) arise from a diverse population of pyramidal neurons. Each type forms local connections with a unique relationship to more superficial layers. In the case of layer 6 neurons, these connections are closely related to layer 4 subdivisions receiving input from different functional streams (Callaway 1998; Sillito et al. 2006).

Organization of the primary visual cortex

The primary visual cortex (V1) consists of six layers (1-6) between the pial surface and the underlying white matter. The principal layer for inputs from the lateral geniculate nucleus (LGN) is layer 4, which is subdivided into four sub layers (4A, 4B, $4 \mathrm{C} \alpha$, and $4 \mathrm{C} \beta$ ), see also Fig. 1. This flow can be described by means of input, intra cortical, and output connections (Kandel et al. 2000):

- Inputs. Axons from magno cellular (M) and parvo cellular (P) cells in the LGN end on spiny stellate cells in the sub layers of $4 \mathrm{C}$, and these cells project axons to layers 2, 3, or 4B. Axons from cell in the intra laminar (I) zones of the LGN project directly to layers 2 and 3.

- Intra cortical connections. Axon collaterals of the pyramidal cell in layers 2 and 3 project to layer 5 pyramidal cells, whose axon collaterals project both to layer 6 pyramidal cells and back to cells in layers 2 and 3. Axon collaterals of layer 6 pyramidal cells then make a loop back to layer 4C onto smooth stellate cells.

Fig. 1 Information flow in the primary visual cortex (V1) based on anatomical connections (Kandel et al. 2000)

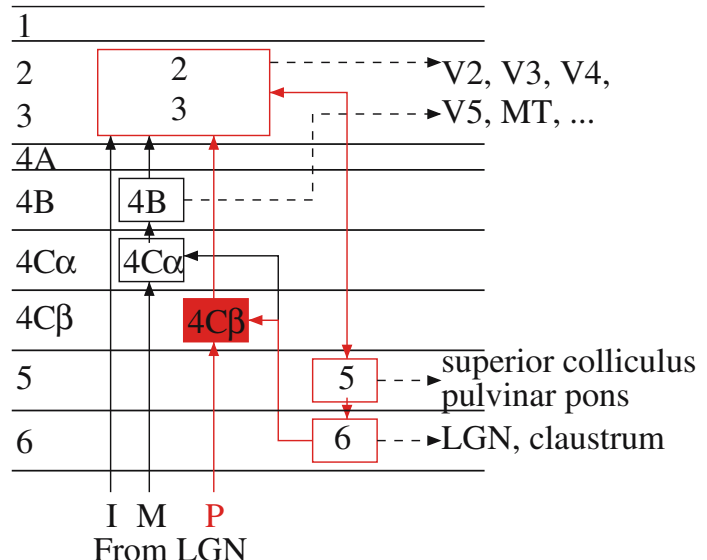

From LGN 
- Outputs. Each layer, except for 4C, has outputs and each is different. Cells in layers 2, 3 , and $4 \mathrm{~B}$ project to extra striate visual cortical areas. Cells in layer 5 project to the superior colliculus, the pons, and pulvinar. Cells in layer 6 project to claustrum and back to the LGN.

The assumption that a $4 \mathrm{C} \beta$ cell receives input from simple (layer 2) or complex cells (layer 3) through layers 5 and 6 makes it plausible that these cells have a far more complex receptive field profile than one can expect from a feed-forward mechanism alone.

Orientation sensitive input responses

In order to model the profiles suggested by Jones et al. (2002), we assume that layer 4C $\beta$ receives complex cell (indirect) input from layers 2, 3, 5, and 6. A computational model of simple and complex cells (Lourens 1998; Würtz and Lourens 2000) is used to form the input of the orientation contrast cells and is introduced only briefly.

The receptive fields of simple cells can be modeled by complex valued Gabor functions:

$$
\widehat{G}_{\sigma, \theta}(x, y)=\exp \left(i \frac{\pi x_{1}}{\sqrt{2} \sigma \lambda}\right) \exp \left(-\frac{x_{1}^{2}+\gamma^{2} y_{1}^{2}}{2 \sigma^{2}}\right)
$$

where $i=\sqrt{-1}, x_{1}=x \cos \theta+y \sin \theta$ and $y_{1}=y \cos \theta-x \sin \theta$. Parameters $\sigma, \lambda, \gamma$, and $\theta$ represent scale, wavelength, spatial aspect ratio, and orientation, respectively. These Gabor functions have been modified, such that their integral vanishes and their one-norm (the integral over the absolute value) becomes independent of $\sigma$, resulting in $G_{\sigma, \theta}(x, y)=\eta \widehat{G}_{\sigma, \theta}(x, y)$, where $\eta=\eta_{\operatorname{Re}}^{+}$for the positive valued real part of $\widehat{G}, \eta=\eta_{\operatorname{Re}}^{-}$for the negative valued real part of $\widehat{G}$, and $\eta=\eta_{\mathrm{Im}}$ for the imaginary part of $\widehat{G}$. For details about these constants see (Lourens 1998). A spatial convolution was used to transform input image $I(x, y)$ by these operators to yield the simple cell operator, and the amplitude of the complex values (Morrone and Burr 1988)

$$
\mathcal{C}_{\sigma, \theta}=\left\|I * G_{\sigma, \theta}\right\|
$$

was taken to obtain the complex cell operator. ${ }^{2}$ This operator forms the basis of the orientation contrast cell operator $\mathcal{O}$ to be described later in this article. A high value at a certain combination of $(x, y)$ and $\theta$ represents evidence for a contour element (bar or edge) oriented orthogonally to $\theta$. Orientations are sampled linearly $\theta_{j}=\pi / N, j=0, \ldots, N-1$, and the scales are sampled $\sigma_{k}=\sigma_{k-2}+\sigma_{k-1}$, for $k=2 \ldots S-1$, where $\sigma_{0}$ and $\sigma_{1}$ represent constants.

\section{Orientation contrast and center orientation preference}

Neuronal cells in area V1 respond to both orientation contrast and center orientation. Depending on the size and orientation of the peripheral patch compared to the preferred orientation of the center patch (which covers the CRF) the response is inhibitory or excitatory. When the patch is similar in size compared to its center patch, the cell tends

\footnotetext{
$\overline{2}$ The preferred orientation $\theta \in[0, \pi)$, since $\mathcal{C}_{\sigma, \theta}=\mathcal{C}_{\sigma, \theta+\pi}$.
} 
to respond strongly to orientation contrast, while a patch that has a diameter of four times the diameter of the central patch tends to respond strongly to the preferred orientation of the central patch (Jones et al. 2002). These findings suggest a varying gain value that depends on the size of the surround patch. This is modeled as follows:

$$
G_{x}(s)=\left[-\frac{2 s^{2}}{30}-\frac{s}{10}+\frac{2}{3}\right]^{\geq 0},
$$

where $s$ denotes the surround patch diameter in degrees, and $[x]^{\geq 0}=x$ if $x \geq 0$ and 0 otherwise. The curve obtained by varying the surround patch diameter is illustrated in Fig. 2a.

The normalized response profile (weight matrix) is modeled as a blend between orientation contrast preference and preferred center orientation:

$$
W\left(c_{p}, c_{o}, c_{s}, s_{o}, s_{s}\right)=G_{x}\left(s_{s} / c_{s}\right) X\left(c_{o}, s_{o}\right)+G_{c} C\left(c_{p}, c_{o}\right),
$$

where $c_{p}, c_{o}$, and $c_{s}$ denote the preferred orientation, used orientation, and diameter of the center patch, all between $0^{\circ}$ and $360^{\circ}$. Likewise $s_{o}$, and $s_{s}$ denote the used orientation and diameter of the surround patch. The normalized orientation contrast profile is as follows:

$$
X\left(c_{o}, s_{o}\right)=\left\{\begin{array}{cl}
0.5-0.5 \cos \left(\frac{\left|c_{o}-s_{o}\right| \pi}{W_{x}}\right) & \text { if } \alpha_{X}\left(c_{o}, s_{o}\right) \leq W_{x} \\
1 & \text { otherwise }
\end{array}\right.
$$

where $W_{x}=90^{\circ}$ is a constant, and

$$
\alpha_{X}\left(c_{o}, s_{o}\right)=\min \left(\left|c_{o}-s_{o}\right|,\left|360+c_{o}-s_{o}\right|,\left|360+s_{o}-c_{o}\right|\right) .
$$

The normalized preferred center orientation is

$$
C\left(c_{p}, c_{o}\right)=\left\{\begin{array}{cl}
0.5 \cos \left(\frac{\left|c_{p}-c_{o}\right| \pi}{W_{c}}\right)+0.5 & \text { if } \alpha_{C}\left(c_{p}, c_{o}\right) \leq W_{c} \\
0 & \text { otherwise }
\end{array}\right.
$$

where $W_{c}=90^{\circ}$ is a constant, and

$$
\alpha_{C}\left(c_{p}, c_{o}\right)=\min \left(\left|c_{p}-c_{o}\right|,\left|360+c_{p}-c_{o}\right|\right)
$$

The response of the $4 \mathrm{C} \beta$ cell as measured by Jones et al. (2002) in Fig. 6 shows a maximum response of around 70 while the minimum response is around 15 . To obtain the response profile as given in Fig. $2 \mathrm{~b}-\mathrm{d}$ the following response was used:

$$
R_{W}=70(W+0.2) \text {. }
$$


a)

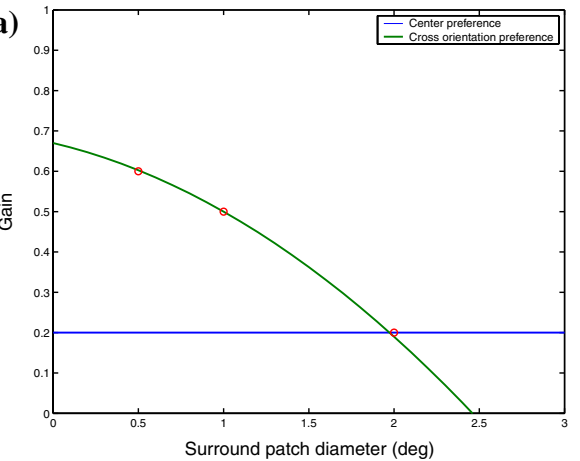

Response Profile $0.5^{\circ}$

c)

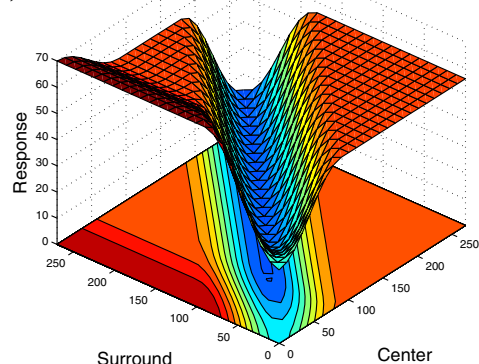

e)
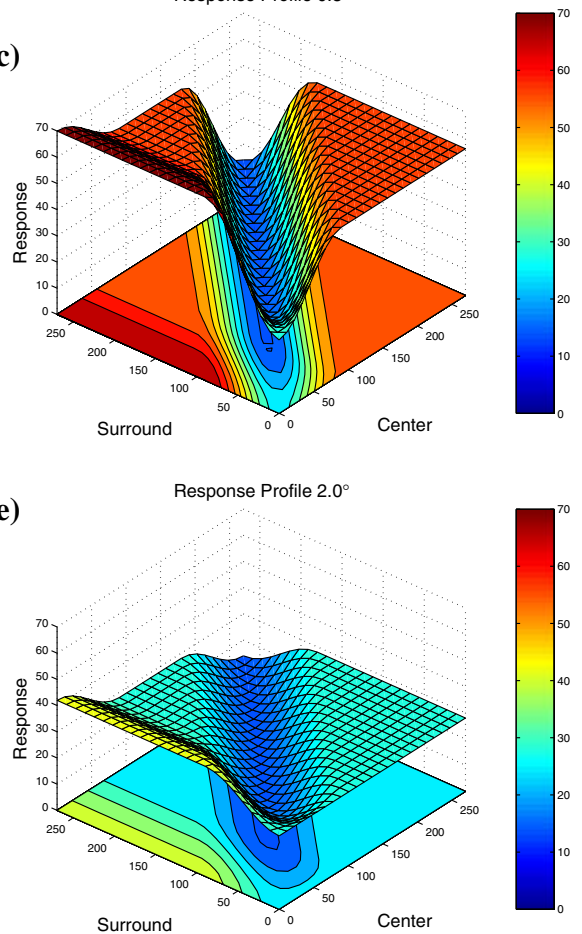

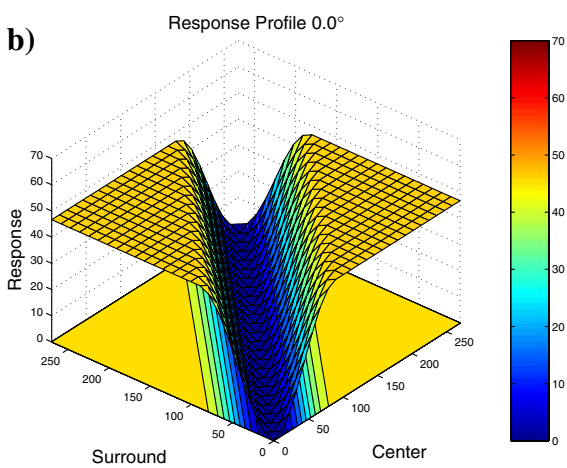

Response Profile $1.0^{\circ}$

d)
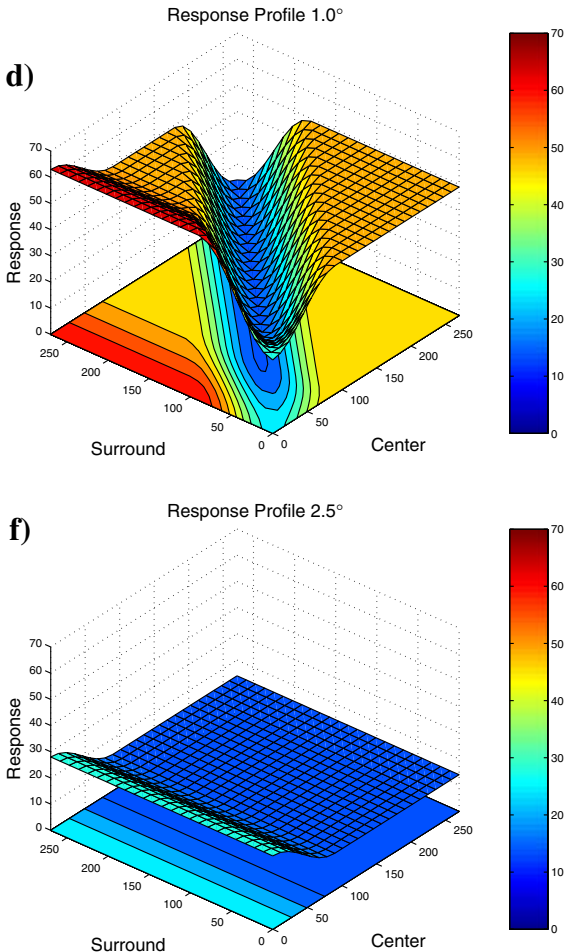

Fig. 2 Modeled response profiles (7) of a non-orientation tuned layer $4 \mathrm{C} \beta$ cells to varying the orientation of both center and surround patch, for a comparison with the measured responses, see Fig. 6 of Jones et al. (2002). (a) Blending curve between orientation contrast and center orientation preference. (b) Modeled profile for $s_{s}=0$, which gives solely a preference to orientation contrast. (c-e) Profiles for $s_{s}=0.5^{\circ}, 1.0^{\circ}$, and $2.0^{\circ}$, respectively. (f) Modeled profile for $s_{s} \geq 2.5$, which solely prefers the center orientation. Parameters used are preferred center orientation $c_{p}=0^{\circ}$, and center radius $c_{s}=0.5^{\circ}$ 
Orientation contrast cell operator

The response of a center patch which covers the CRF is obtained as follows:

$$
\mathrm{C}_{\sigma, c_{s}}=\mathcal{C}_{\sigma, \theta_{i}} * g_{c_{s} / 6}
$$

where $\theta_{i}=i \pi / N, i=0, \ldots, N$, and

$$
g_{\sigma}(x, y)=\frac{\exp \left(-\frac{x^{2}+y^{2}}{2 \sigma^{2}}\right)}{2 \pi \sigma^{2}}
$$

is a $2 \mathrm{D}$ Gaussian function.

The response of a surround patch is obtained by taking the maximum response of differently sized surround patches

$$
\mathrm{S}_{\sigma, c_{s}, S_{\min }, S_{\max }, Q}=\max _{(x 1, y 1)}\left(W\left(c_{p}, c_{o}, c_{s}, s_{o}, s_{s}\right)-W_{s}\right) \max _{j}\left(\widehat{\mathcal{C}}_{\sigma, \theta_{i}}\left(x_{1}, y_{1}\right)\right),
$$

where

$$
\begin{array}{r}
\widehat{\mathcal{C}}_{\sigma, \theta_{i}}=\mathcal{C}_{\sigma, \theta_{i}} * g_{s_{s_{j}} / 6} \\
s_{s_{j}}=s_{\min }+j \frac{s_{\max }-s_{\text {min }}}{Q-1}
\end{array}
$$

has a linearly increasing patch size between $s_{\min }$ and $s_{\max }, j=0, \ldots, Q-1$, and $Q$ is the number of surround patch sizes. Let $j_{\max }$ denote the index $j$ for which holds $\widehat{\mathcal{C}}_{\sigma, \theta_{i}}$ is maximal. Weight $W$ from (4) is in the $0^{\circ}-90^{\circ}$ range, since we assume that the grating pattern is static rather than moving in a specific direction, $W_{s}$ is an inhibitive weight, and $(x 1, y 1)$ are the spatial positions of the outer stimulus. Since these patches largely overlap resampling is used to reduce computational time. Preferred center orientation $c_{p}$, center orientation $c_{o}$, surround orientation $s_{o}$, and surround patch size $s_{s}$ are as follows:

$$
\begin{gathered}
c_{p}=c_{o}= \begin{cases}180 i / N & \text { if } i \leq N / 2 \\
180(N-i) / N & \text { otherwise }\end{cases} \\
s_{s}=s_{\min }+j_{\max } \frac{s_{\max }-s_{\min }}{Q-1} \\
s_{o}= \begin{cases}180 j_{\max } / N & \text { if } j_{\max } \leq N / 2 \\
180\left(N-j_{\max }\right) / N & \text { otherwise }\end{cases}
\end{gathered}
$$

The orientation contrast operator which comprises a center response and a surround response that depends on the center response is as follows:

$$
\mathcal{O}_{\sigma, c_{s}, S_{\min }, s_{\max }, Q}=\left(\mathrm{C}_{\sigma, c_{s}}+w \mathrm{~S}_{\sigma, c_{s}, s_{\min }, s_{\max }, Q}\right) * g_{c_{s} / 6},
$$

where weight $w=\mathrm{C}_{\sigma, c_{s}} / R$ is a weight that is dependent on the center response C. In all simulations constant $R=255$ was used to bound $w$ between 0 and 1 . 


\section{Responses to test patterns}

The input stimuli used in the simulation have a center radius of 24 pixels and surround radii of 24 (Fig. 3a), 48, 72 (Fig. 3b), or 96 pixels. The block gratings consist of alternating black and white bars which are both 8 pixels wide. A complex cell operator $\mathcal{C}_{\sigma, \theta}$ with
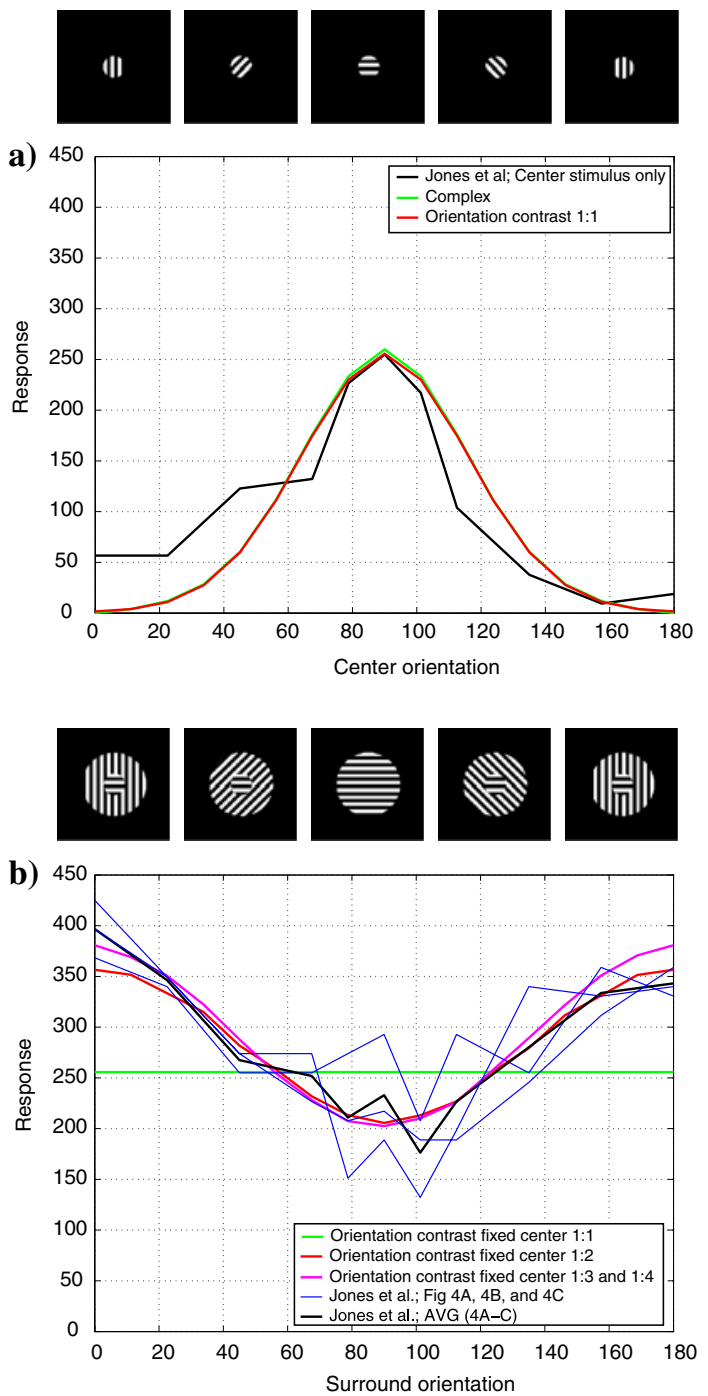

Fig. 3 Response characteristics of orientation contrast sensitive cells, see Fig. 4A-F from Jones et al. (2002). (a) Input stimuli with preferred orientations of $0^{\circ}, 45^{\circ}, 90^{\circ}, 135^{\circ}$, and $180^{\circ}$, and below the response profiles to these stimuli of the measured V1 cells, complex cells $(\mathcal{C}$-operator) and orientation contrast type of cell ( $\mathcal{O}$-operator). (b) Input stimuli with surround, with preferred center orientation of $90^{\circ}$ and varying surround orientation from left to right from $0^{\circ}$ to $180^{\circ}$. The ratio between center and surround of these stimuli is 1:3 (top). Response profiles for measured cells and center-only (1:1), and center-surround (1:2, $1: 3$, and 1:4) stimuli. Responses have been normalized to the maximum response of the modeled complex cells (255). The inhibitory weight $W_{s}=0.45$ which yields similar response profiles as the measured V1 cells 


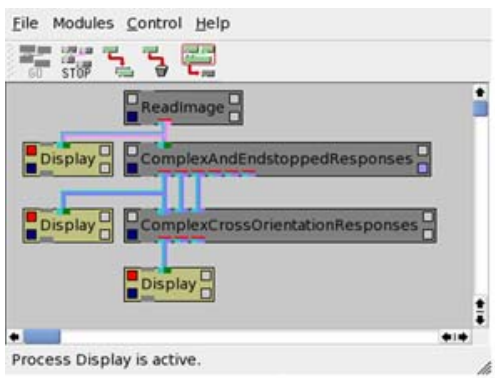

a)

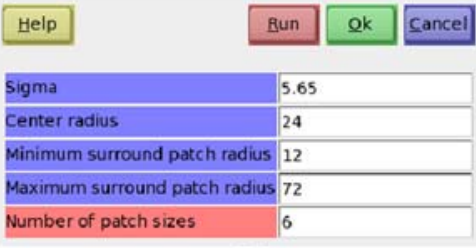

b)

Fig. 4 (a) TiViPE network. (b) Parameters used for cross-orientation type of cells

$\sigma=4 \sqrt{2}$ and an orientation $\theta$ corresponding to the preferred orientation of the grating pattern yields an optimal response (255), see also Fig. 3a, for the complex cell operator $\mathcal{C}$ in the center of the input stimuli of Fig. $3 a$ and $b$. When the center-only input stimulus is applied to orientation contrast operator $(\mathcal{O})$ for the preferred horizontal center orientation the $\mathcal{O}$-operator has a very similar response profile compared to the $\mathcal{C}$-operator, but where the results of $\mathcal{C}$-operator remain the same, the $\mathcal{O}$-operator is influenced by its surround as illustrated in Fig. 3b ("Orientation contrast fixed center 1:3"). The profile is very similar to the one given by Jones et al. (2002).

The orientation contrast cell operator $\mathcal{O}$ from (13) has been implemented in visual programming environment TiViPE (Lourens 2004) (Fig. 4). The orientation contrast simulation that is represented by a network of connected icons consists of a "ReadImage" icon which generates the input stimulus, its connected "Display" icon yields the images provided in Fig. $3 \mathrm{a}$ and b. The "ComplexAndEndstopppedResponse", produced the responses of the $\mathcal{C}$-operator (2). Its output forms the input of the "ComplexCrossOrientationResponses" and gives the responses of the $\mathcal{O}$-operator (13). The values at the center of the two other "Display" icons have been used to construct Fig. 3c.

\section{The role of orientation contrast cells}

Orientation contrast sensitive cells may play a key role in intermediate-level visual tasks, such a perceptual pop-out (Nothdurft et al. 1999), contextual modulation (Levitt and Lund 1997; Das and Gilbert 1999; Dragoi and Sur 2000), and junction detection (Sillito et al. 1995; Das and Gilbert 1999; Jones et al. 2002).

A simple image (first column of Fig. 5a) reveals the properties of the orientation contrast operator proposed in (13). The operator enhances the responses at edges near junctions and decreases the responses at edges otherwise (first column of Fig. 5b). If the operator would be applied in a repetitive manner, responses appear only at the edges near junctions, suggesting that they might play a key role in junction detection.

Orientation contrast responses to the bottle image (second column of Fig. 5a) also demonstrates that the background at the bottom, left, and right of the bottle is enhanced suggesting that figure-ground segregation can be facilitated. However, neither the response profile in the second column of Fig. $5 b$ nor the difference between orientation-contrast and complex cell responses demonstrate perceptual pop-out effects. 

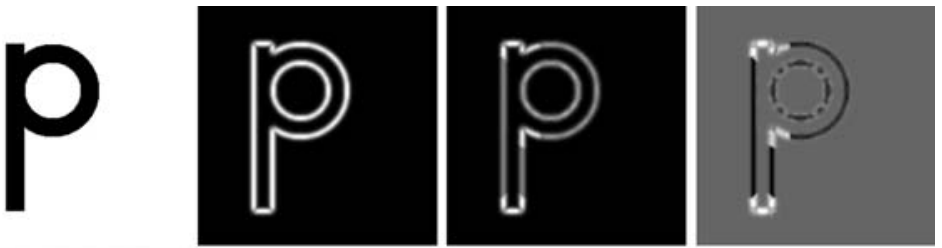

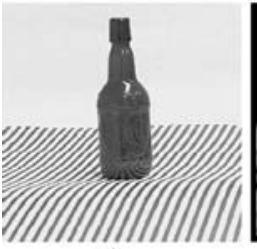

a)

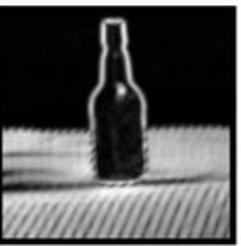

b)

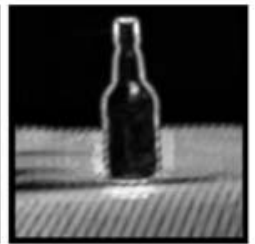

c)

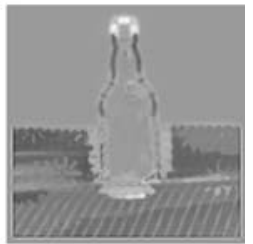

d)

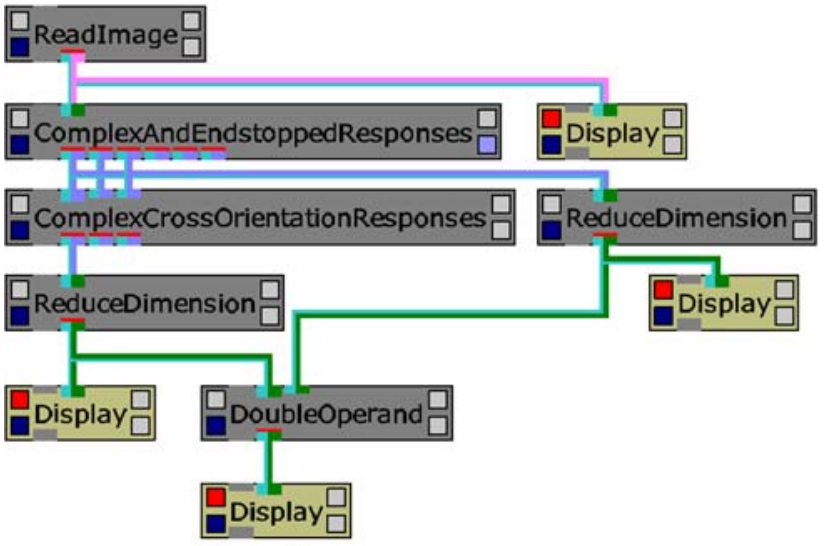

e)

Fig. 5 (a) Input images. (b) Normalized complex cell responses. (c) Normalized orientation contrast responses. (d) Normalized difference in response between complex and orientation contrast responses. White denotes stronger, gray equal, and black weaker response of the orientation contrast cell operator compared to the complex cell operator. (e) TiViPE simulation. Top, middle, bottom left, and bottom right "Display", icons provide the results given in $(\mathbf{a}),(\mathbf{b}),(\mathbf{c})$, and (d), respectively. The used parameters are $\sigma=2.82$, a center radius of 4 , minimum and maximum surround patch radii of, respectively, 2 and 12 , six different patch sizes, and images of $256 \times 256$ pixels

\section{Discussion}

Many neurons in primary visual cortex (V1) respond differently to a simple visual element presented in isolation compared to when it is embedded in a more complex stimulus. Typically the surround influence was suppressive when the surround grating was at the neuron's preferred orientation (Cavanaugh et al. 2002), but when the orientation in the surround was perpendicular to the preferred orientation facilitation became evident (Sillito et al. 1995; Shevelev et al. 1998; Cavanaugh et al. 2002; Jones et al. 2002). The difference is in the modulation by surrounding elements, hence it could provide neurons with a graded specialization for processing junctions (Sillito et al. 1995; Das and Gilbert 1999). These neurons also respond to a grating or a single bar of a preferred orientation and are in that respect too general to be purely responding to junctions. In the monkey the majority of cells showed response suppression with increasing grating patch diameter (Born and 
Tootell 1991; Sillito et al. 1995) therefore it is likely that a group of these neurons responds to junctions and facilitates pop-out patterns (Knierim and van Essen 1992; Nothdurft et al. 1999).

The proposed model for orientation contrast cells uses complex cell input that is provided by the indirect pathway from layers $2,3,5$, and 6 of V1. It is therefore likely that the layer 6 to 4 projection acts as a feedback connection as has been suggested by Callaway (1998). The model yields appropriate characteristics to test patterns as used by Jones et al. (2002) and hence provides evidence that orientation contrast effects in layer 4C $\beta$ can be adequately explained on the basis of this "feedback" connectivity. This is an issue that has not yet been addressed experimentally in primate V1, because it may not be realistic to carry out controlled experiments to test this hypothesis. Experimental studies in the cat, exploring the role of the corticogeniculate projection from V1 to the lateral geniculate nucleus, provide indirect supporting evidence for this type of feedback. Thus, relay cells in cat lateral geniculate nucleus, that are themselves non-oriented, have also been shown to exhibit orientation contrast effects (Sillito et al. 1993; Sillito and Jones 2002) and these effects were shown to depend on the feedback projection from cortical V1 layer 6 cells.

The current model provides an initial basis for orient contrast type of cells, but Jones et al. (2002) provided more experimental results that could lead to a set of orientationcontrast models with an extended set of properties. The model has not yet been evaluated with natural stimuli, or test stimuli that differ in contrast only. Latter is attractive since the spatial summation properties of most V1 cells vary with stimulus contrast (Sceniak et al. 1999). Future work will include the integration of the model itself into a highly parallel vision system that can be used in intelligent visual devices or a robot in a real world environment.

\section{References}

Born RT, Tootell RBH (1991) Single unit and 2-deoxyglucose studies of side inhibition in macaque striate cortex. Proc Natl Acad Sci USA 88:7071-7075

Callaway EM (1998) Local circuits in primary visual cortex of the macaque monkey. Annu Rev Neurosci 21:47-74

Cavanaugh JR, Bair W, Movshon JA (2002) Selectivity and spatial distribution of signals from the receptive field surround in macaque V1 neurons. J Neurophysiol 88:2547-2556

Das A, Gilbert CD (1999) Topography of contextual modulations mediated by short-range interactions in primary visual cortex. Nature 399:655-661

Dragoi V, Sur M (2000) Dynamic properties of recurrent inhibition in primary visual cortex: contrast and orientation dependece of contextual effects. J Neurophysiol 83:1019-1030

Jones HE, Wang W, Sillito AM (2002) Spatial organization and magnitude of orientation contrast interactions in primate V1. J Neurophysiol 88:2797-2808

Kandel ER, Schwartz JH, Jessell TM (2000) Principles of neural science, 4th edn. Mc Graw Hill

Knierim JJ, van Essen DC (1992) Neuronal responses to static texture patterns in area V1 of the alert macaque monkey. J Neurophysiol 67(5):961-980

Levitt JB, Lund JS (1997) Contrast dependence of contextual effects in primate visual cortex. Nature 387:73-76

Lourens T (1998) A biologically plausible model for corner-based object recognition from color images. Shaker Publishing B.V., Maastricht The Netherlands

Lourens T (2004) TiViPE-Tino's visual programming environment. In: The 28th Annual International Computer Software \& Applications Conference, IEEE COMPSAC 2004, pp 10-15

Morrone MC, Burr DC (1988) Feature detection in human vision: a phase-dependent energy model. Proc. Roy Soc Lond 235:335-354

Nothdurft HC, Gallant JL, Essen DCV (1999) Response modulation by texture surround in primate area V1: correlates of "popout' under anesthesia. Visual Neurosci 16:15-34

Sceniak MP, Ringach DL, Shapley R (1999) Contrast's effect on spatial summation by macaque V1 neurons. Nature Neurosci 2(8):733-739 
Shevelev IA, Lazareva NA, Sharaev GA, Novikova RV, Tikhomirov AS (1998) Selective and invariant sensitivity to crosses and corners in cat striate neurons. Neuroscience 84(3):713-721

Sillito AM, Cudeiro J, Jones HE (2006) Always returning: feedback and sensory processing in visual cortex and thalamus. Trends Neurosci 29(6):307-316

Sillito AM, Cudeiro J, Murphy PC (1993) Orientation sensitive elements in the corticofugal influence on centre-surround interactions in the dorsal lateral geniculate nucleus. Exp Brain Res 1:6-16

Sillito AM, Grieve KL, Jones HE, Cudiero J, Davis J (1995) Visual cortical mechanisms detecting focal orientation discontinuities. Nature 378:492-496

Sillito AM, Jones HE (2002) Corticothalamic interactions in the transfer of visual information. Philos Trans R Soc Lond B Biol Sci 357(1428):1739-1752

Würtz RP, Lourens T (2000) Corner detection in color images through a multiscale combination of endstopped cortical cells. Image Vision Comput 18(6-7):531-541

Yu C, Klein SA, Levi DM (2002) Facilitation of contrast detection by cross-oriented surround stimuli and its psychophysical mechanisms. J Vision 2:243-255 